\title{
Article
}

\section{Oral Anticoagulant Adequacy in Non-Valvular Atrial Fibrillation in Primary Care: A Cross-Sectional Study Using Real-World Data (Fantas-TIC Study)}

\author{
M. Rosa Dalmau Llorca ${ }^{1,2,3,+} \mathbb{C}^{\mathbb{C}}$, Carina Aguilar Martín ${ }^{3,4,5, \dagger}$, Noèlia Carrasco-Querol ${ }^{3,4, * \mathbb{C}}$, \\ Zojaina Hernández Rojas 1,2,3, Emma Forcadell Drago ${ }^{1,3}$, Dolores Rodríguez Cumplido ${ }^{3,6}$, \\ Josep M. Pepió Vilaubíi ${ }^{1,3}$, Elisabet Castro Blanco ${ }^{2,3}$, Alessandra Q. Gonçalves ${ }^{3,4,7, \dagger}$ \\ and José Fernández-Sáez ${ }^{2,3,4,+}$
}

check for

updates

Citation: Dalmau Llorca, M.R.;

Aguilar Martín, C.; Carrasco-Querol,

N.; Hernández Rojas, Z.; Forcadell

Drago, E.; Rodríguez Cumplido, D.;

Pepió Vilaubí, J.M.; Castro Blanco, E.;

Gonçalves, A.Q.; Fernández-Sáez, J.

Oral Anticoagulant Adequacy in

Non-Valvular Atrial Fibrillation in

Primary Care: A Cross-Sectional

Study Using Real-World Data

(Fantas-TIC Study). Int. J. Environ.

Res. Public Health 2021, 18, 2244.

https://doi.org/10.3390/

ijerph18052244

Received: 14 January 2021

Accepted: 16 February 2021

Published: 24 February 2021

Publisher's Note: MDPI stays neutral with regard to jurisdictional claims in published maps and institutional affiliations.

Copyright: (c) 2021 by the authors. Licensee MDPI, Basel, Switzerland. This article is an open access article distributed under the terms and conditions of the Creative Commons Attribution (CC BY) license (https:/ / creativecommons.org/licenses/by/ $4.0 /)$
1 Equip d'Atenció Primària Terres de l'Ebre, Institut Català de la Salut, Tortosa, 43500 Tarragona, Spain; rdalmau.ebre.ics@gencat.cat (M.R.D.L.); zojahernandez@gmail.com (Z.H.R.); eforcadellg.ebre.ics@gencat.cat (E.F.D.); jmpepio.ebre.ics@gencat.cat (J.M.P.V.)

2 Grupo GAVINA, Campus Terres de l'Ebre, Universitat Rovira i Virgili, Tortosa, 43500 Tarragona, Spain; elicasblan@gmail.com

3 GAVINA Research Group, Tortosa, 43500 Tarragona, Spain; caguilar.ebre.ics@gencat.cat (C.A.M.); aqueiroga.ebre.ics@gencat.cat (A.Q.G.); jfernandez@idiapjgol.info (J.F.-S.); drfif@gmail.com (D.R.C.)

4 Unitat de Suport a la Recerca Terres de l'Ebre, Fundació Institut Universitari per a la Recerca a l'Atenció Primària de Salut Jordi Gol i Gurina (IDIAPJGol), Tortosa, 43500 Tarragona, Spain

5 Unitat d'Avaluació, Direcció d'Atenció Primària Terres de l'Ebre, Institut Català de la Salut, Tortosa, 43500 Tarragona, Spain

6 Hospital Universitari de Bellvitge, Institut Català de la Salut, 08907 Barcelona, Spain

7 Unitat Docent de Medicina de Familia i Comunitària, Tortosa-Terres de l'Ebre, Institut Català de la Salut, Tortosa, 43500 Tarragona, Spain

* Correspondence: ncarrasco.querol@gmail.com

† Co-senior authors: Dalmau Llorca MR, Aguilar Martín C, Gonçalves AQ and Fernández-Sáez. M. Rosa Dalmau Llorca is the Principal Investigator in this study.

Abstract: Background: Oral anticoagulants (OAs) are the treatment to prevent stroke in atrial fibrillation (AF). Anticoagulant treatment choice in non-valvular atrial fibrillation (NVAF) must be individualized, taking current guidelines into account. Adequacy of anticoagulant therapy under the current criteria for NVAF in real-world primary care is presented. Methods: Cross-sectional study, with real-world data from patients treated in primary care (PC). Data were obtained from the System for the Improvement of Research in Primary Care (SIDIAP) database, covering 60,978 NVAFanticoagulated patients from 287 PC centers in 2018. Results: In total, 41,430 (68\%) were treated with vitamin K antagonists (VKAs) and 19,548 (32\%) NVAF with direct-acting oral anticoagulants (DOACs). Inadequate prescription was estimated to be $36.0 \%$ and $67.6 \%$, respectively. Most DOAC inadequacy $(77.3 \%)$ was due to it being prescribed as a first-line anticoagulant when there was no history of thromboembolic events or intracranial hemorrhage (ICH). A total of $22.1 \%$ had missing estimated glomerular filtration rate (eGFR) values. Common causes of inadequate VKA prescription were poor control of time in therapeutic range (TTR) $(98.8 \%)$ and $\mathrm{ICH}(2.2 \%)$. Conclusions: Poor adequacy to current criteria was observed, being inadequacy higher in DOACs than in VKAs. TTR and GFR should be routinely calculated in electronic health records (EHR) to facilitate decisionmaking and patient safety.

Keywords: atrial fibrillation; direct oral anticoagulants; renal function; time in therapeutic range; vitamin $\mathrm{K}$ antagonists

\section{Introduction}

Oral anticoagulants (OAs) are used to prevent stroke in atrial fibrillation (AF). The American guidelines recommend using warfarin, a vitamin $\mathrm{K}$ antagonist (VKA), and direct- 
acting oral anticoagulants (DOACs) to prevent stroke in AF in patients with CHA2DS2VASC $\geq 2$, taking into account individual risk/benefit of bleeding [1]. The European Society of Cardiology guidelines recommend the use of DOACs as first-line treatment for non-valvular atrial fibrillation (NVAF) [2].

In Catalonia, anticoagulant therapy for NVAF follows the "Informe de Posicionamiento terapéutico" (IPT) (Therapeutic Positioning Report) and the "Pautes per a l'harmonització del tractament" (PHT) (Therapeutic Harmonization Guidelines for the Use of Oral Anticoagulants) [3,4] from the Ministry of health, social services and equality of Spain and the Catalan Health Service, respectively. Under these guidelines, VKA are the first-line treatment for $\mathrm{AF}$ cases requiring anticoagulation, while DOAC are used in specific situations of NVAF [4].

The various DOACs are at least as effective as warfarin at preventing stroke in NVAF. The four DOACs (dabigatran, rivaroxaban, apixaban, and edoxaban) commercialized up to 2017 in Spain, and currently in many European countries, reduce the rates of stroke, systemic embolism, major bleeding, intracranial hemorrhage (ICH), cardiovascular mortality and total mortality, but are associated with a higher risk of intestinal bleeding [5-8]. Despite the dosage and interaction advantages of DOACs over VKAs, physicians individually evaluate the choice of anticoagulant [3,4]. Since VKAs remain the first-line treatment for NVAF in Spain, prescription of DOACs in Spain is amongst the lowest in Europe [9], although DOAC prescription is currently increasing. However, there is a trend towards inadequate OA prescription in NVAF patients that was not yet sufficiently described with population data [10].

This study analyzes the adequacy of the anticoagulant therapy prescription (VKAs and DOACs) in NVAF under the current recommendation criteria in primary care (PC) Catalan population, using real-world health care data.

\section{Material and Methods}

\subsection{Study Design}

A cross-sectional study with real-world data of primary care patients of the Catalan Institute of Health (ICS) was conducted. The study included patients with NVAF diagnoses in 2018 at the 287 ICS primary care centers (PCCs). These PCCs are responsible for the care of an estimated 5,564,292 people ( $80 \%$ of the Catalan and $>10 \%$ of the Spanish populations), and employ 3384 physicians.

\subsection{Data Source}

Data were obtained from the SIDIAP (Information System for Research in Primary Care) population database, which is representative of the Catalan population [11,12]. We identified 97,350 patients in the SIDIAP with a diagnosis of AF for at least 12 months (Figure 1). Patients with an active prescription for an anticoagulant on 1 January 2018 were included. We considered all authorized anticoagulant treatments with VKAs (acenocoumarol and warfarin) and DOACs (dabigatran, rivaroxaban, apixaban, and edoxaban) in Spain in 2016. Drug data based on Anatomic Therapeutic Chemical (ATC) codes were collected [13].

SIDIAP contains anonymized clinical information from various data sources [11,12]: (1) electronic health records (EHRs) from ICS primary care (known as eCAP_Estació Clinica d'Atenció Primària), which, since 2006, has included information on sociodemographic characteristics, health conditions registered as International Classification of Diseases (ICD) 10 codes [14], general practitioner prescriptions and clinical parameters; (2) laboratory data; (3) prescription data, available since 2005, with information on all pharmaceutical products dispensed by community pharmacies of the Catalan Health System, based on ATC classification system codes [13]. 


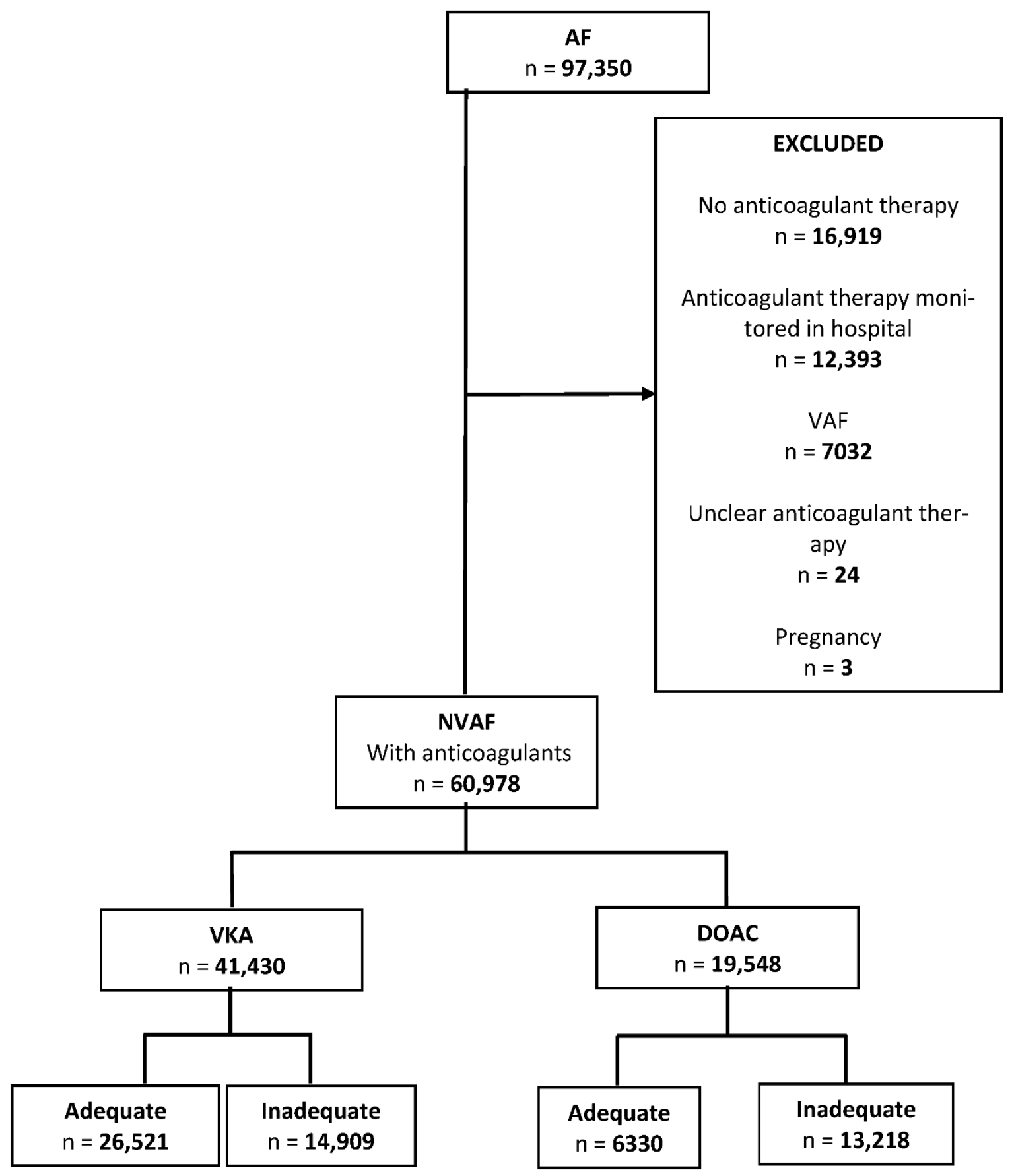

Figure 1. Flowchart of the study.

\section{Study Population}

Inclusion and Exclusion Criteria

We included patients who received OA treatment and monitored the anticoagulant therapy in PCCs of the ICS, diagnosed with NVAF one year before the study date, and with at least six controls of the International Normalized Ratio (INR) during the year before the study. This restriction minimizes INR variability at the start of the treatment and avoids the effect of temporary withdrawal of VKAs in patients with good control of INR.

Patients were considered to have been exposed to anticoagulation if they were prescribed anticoagulants (acenocoumarol, warfarin, dabigatran, rivaroxaban, apixaban, or 
edoxaban) for at least 2 months before the study date. The anticoagulant medication included in the study was those started the closest to the study date.

We excluded patients with no OA therapy, patients whose OA therapy was monitored in hospital, those with valvular atrial fibrillation (mitral stenosis or with a mechanical prosthetic valve), pregnant women, and patients whose anticoagulant treatment at the beginning of the study could not be ascertained (Figure 1).

\section{Study Variables}

Main variable. Adequacy of anticoagulant treatment (VKAs and DOACs) under IPT criteria (Table 1).

Table 1. Catalan (PTH) and IPT Spanish (IPT) criteria for oral anticoagulant therapy.

VKAs (acenocoumarol and warfarin) are the first-line therapy in patients newly diagnosed with NVAF (except for patients meeting criteria for DOACs)

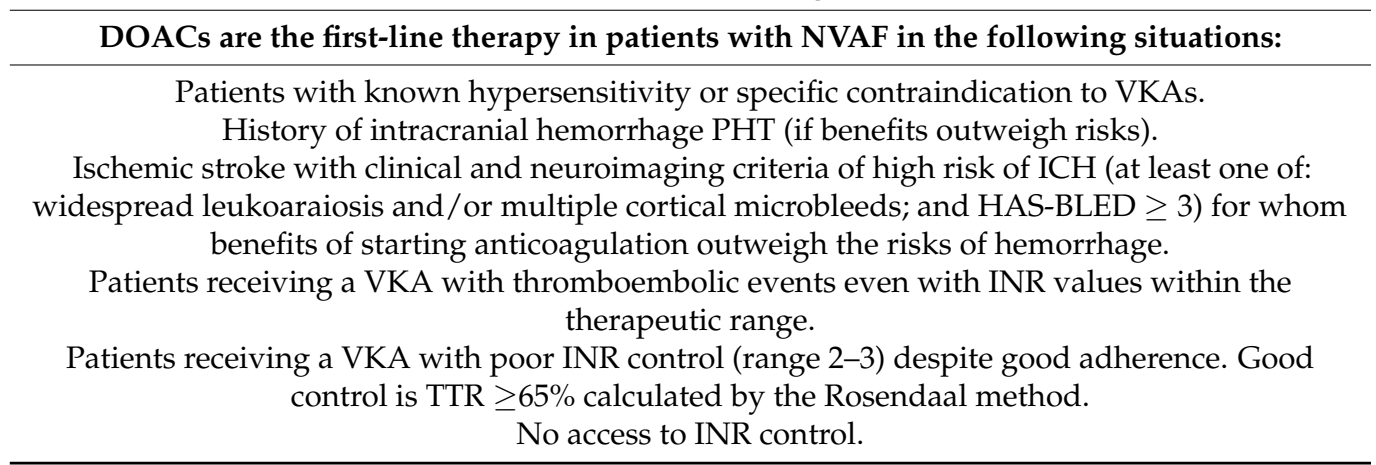

Abbreviations: IPT: Therapeutic Positioning. Report UT_DAOA/V5/211122016; PHT: Guidelines for the Therapeutic Harmonization in the use of oral anticoagulants with Atrial Fibrillation; VKA: vitamin K antagonist; DOACs: direct oral anticoagulants; NVAF: non-valvular atrial fibrillation; INR: International Normalized Ratio; TTR: time in therapeutic range; ICH: intracranial hemorrhage; HAS-BLED: bleeding risk score.

Adequacy of VKA prescription. Patients receiving a VKA who met one of the following conditions [4]: $\mathrm{TTR}^{\mathrm{R}} \geq 65 \%$ (according to Rosendaal's formula [15]), $\mathrm{TTR}^{\mathrm{R}}<65 \%$ and glomerular filtration (eGFR estimated using CKD-EPI) $<15 / \mathrm{min} / 1.73 \mathrm{~m}^{2}$, history of adverse drug reaction (ADR) to DOACs (including allergy), a missing value of ${ }_{e} G F R$ or TTR ${ }^{R}$. Inadequate prescription of VKAs was concluded for patients receiving a VKA who met one of the following conditions: history of ADR to VKAs or intracranial hemorrhage (ICH) with $\mathrm{e}$ GFR $>15 / \mathrm{min} / 1.73 \mathrm{~m}^{2}$.

Adequacy of DOAC prescription. Patients who received one DOAC and met one of the following conditions [4]: pre-DOAC TTR ${ }^{\mathrm{R}}<65 \%$ if the first OA was a VKA, or ADR to VKA, thromboembolic event (TEE) after starting VKA with TTR $\geq 65 \%$ (post-VKA TTE), $\mathrm{ICH}$ or stroke with a high risk of bleeding (SHRB). Inadequate prescription of DOACs was concluded for patients receiving DOACs who met one of the following conditions: ADR to DOAC, eGFR $<15 / \mathrm{min} / 1.73 \mathrm{~m}^{2}$, or a missing eGFR value, or when the DOAC was prescribed as first-line anticoagulant with no history of post-VKA TTE, SHRB or ICH.

Secondary variables. Sociodemographic variables, type of anticoagulant treatment, place of OA prescription, history of cardiovascular disease (CVD), ICH, morbidity, gastrointestinal hemorrhage $(\mathrm{GH})$, history of high risk of bleeding (HRB) and other hemorrhages, estimated scores [15-18] based on participants' real world data (Table 2) and calculated constructed variables (see Table 3). Diseases were classified as specified in the ICD10 code list [14]. 
Table 2. Scores evaluated in the present study.

\begin{tabular}{|c|c|c|}
\hline Score & Definition & Reference \\
\hline $\mathrm{CHA}_{2} \mathrm{DS}_{2}$-VASC & $\begin{array}{l}\text { Score based on having or not having diagnosed heart failure (HF) } \\
\text { and/or Teichholz ejection fraction }<40 \%, H T \text {, DM, stroke, IC or } \\
\text { PA, or aortic atherosclerosis, age between } 65 \text { and } 74 \text { or }>75 \text { years. } \\
\text { Annual scores from } 2011 \text { to } 2017 \text { were obtained. }\end{array}$ & [16] \\
\hline HAS-BLED & $\begin{array}{l}\text { Score based on having or not having systolic blood pressure }>160 \\
\text { mmHg, kidney failure or CKD-EPI }<45 \text { in two consecutive } \\
\text { measurements, LF, stroke or ICH, HRB, Rosendaal TTR }<65 \% \text {. } \\
\text { Annual scores from } 2011 \text { to } 2017 \text { were obtained. }\end{array}$ & [17] \\
\hline TTR & $\begin{array}{l}\text { Calculated by the Rosendaal method, which yields the percentage } \\
\text { of the time the patient is within the } 2-3 \text { range, assuming a linear } \\
\text { progression between the consecutive INR values and calculating } \\
\text { the daily specific INR. Poor control is concluded when TTR }<65 \% \text {. } \\
\text { Two annual values of } 12 \text { and } 6 \text { months (last months of the year) } \\
\text { from } 2011 \text { to } 2017 \text { were obtained. }\end{array}$ & [15] \\
\hline CKD-EPI $\left(\mathrm{mL} / \mathrm{min} / 1.73 \mathrm{~m}^{2}\right)$ & $\begin{array}{l}\text { eGFR is based on creatinine and the Chronic Kidney Disease } \\
\text { Epidemiology Collaboration equation (CKD-EPI). Annual values } \\
\text { from } 2011 \text { to } 2017 \text { were obtained if there was a creatinine value } \\
\text { that year, otherwise it was considered a missing value. If there } \\
\text { was more than one creatinine value per year, the lowest value } \\
\text { was considered. }\end{array}$ & [18] \\
\hline
\end{tabular}

HT: hypertension; DM: diabetes mellitus; IC: ischemic cardiomyopathy; PA: peripheral arteriopathy; LF: liver failure; HRB: high risk of bleeding; INR: International Normalized Ratio; TTR: time in therapeutic range according to the Rosendaal method; eGFR: glomerular filtration according to CKD-EPI $\left(\mathrm{mL} / \mathrm{min} / 1.73 \mathrm{~m}^{2}\right)$ score; ICH: intracranial hemorrhage.

Table 3. Definition of the variables evaluated in the present study.

\begin{tabular}{|c|c|}
\hline Variable & Definition \\
\hline Thromboembolic event (TTE) & $\begin{array}{c}\text { Includes IC, PA, aortic atherosclerosis, ischemic stroke, } \\
\text { including TIA }\end{array}$ \\
\hline $\begin{array}{l}\text { Thromboembolic event after starting VKA with TTR } \geq 65 \% \\
\text { (post-VKA TTE) }\end{array}$ & $\begin{array}{c}\text { Includes IC, PA, aortic atherosclerosis, ischemic stroke, } \\
\text { including TIA, after starting a VKA }\end{array}$ \\
\hline Stroke with high risk of bleeding (SHRB) & $\begin{array}{l}\text { Includes ischemic stroke, including TIA in patients with } \\
\text { HASBLED } \geq 3 \text { or HRB }\end{array}$ \\
\hline TTR of 6 or 12 months (TTR) & $\begin{array}{l}\text { We calculated TTR for the } 12 \text { months before the cut-off date. If } \\
\text { there was no measurement, we obtained that for } 6 \text { months }\end{array}$ \\
\hline eGFR & $\begin{array}{l}\text { Glomerular filtration in } \mathrm{mL} / \mathrm{min} / 1.73 \mathrm{~m}^{2} \text {, according to the } \\
\text { Chronic Kidney Disease Epidemiology Collaboration equation } \\
\text { (CKD-EPI) [18] for the previous } 12 \text { months, or for the lowest } \\
\text { creatinine measurement during the previous } 24 \text { months. } \\
\text { Categorized as } \geq 15 \mathrm{~mL} / \mathrm{min} / 1.73 \mathrm{~m}^{2} \text { or }<15 \mathrm{~mL} / \mathrm{min} / 1.73 \mathrm{~m}^{2}\end{array}$ \\
\hline Adverse drug reaction (ADR) & $\begin{array}{l}\text { For VKAs and DOACs, includes moderate and severe adverse } \\
\text { reactions and allergies to the medication group, without } \\
\text { specifying the active ingredient }\end{array}$ \\
\hline TTR before starting DOAC (pre-DOAC TTR) & $\begin{array}{l}\text { We calculated TTR before starting DOAC administration in } \\
\text { patients who had taken VKAs and categorized values } \\
\text { as } \geq 65 \% \text { or }<65 \%\end{array}$ \\
\hline First oral anticoagulant (First OA) & $\begin{array}{l}\text { The first OA prescribed was determined in patients who had } \\
\text { taken VKAs or DOACs }\end{array}$ \\
\hline No event & No post-VKA TTE, no ICH, no SHB \\
\hline
\end{tabular}

VKA: vitamin K antagonist; DOACs: direct oral anticoagulants; IC: ischemic cardiomyopathy; PA: peripheral arteriopathy; TIA: transient ischemic attack; HRB: high risk of bleeding; TTR: time in therapeutic range; eGFR: estimated glomerular filtration; ICH: intracranial hemorrhage. 


\section{Statistical Analysis}

Anonymized data were exported from the SIDIAP database to the Statistical Package of Social Sciences IBM (SPSS) version 20.0. As this was a population-based study, the sample guaranteed $\geq 99 \%$ statistical power. Data were cleaned, taking into account the minimum and maximum values of variables, and an analysis of missing values was carried out. Variables were summarized as the mean of frequencies and percentages for categorical variables, and the median and interquartile range for continuous variables. Variables were created to describe adequacy according to IPT criteria [4] (Table 1). Two-proportion Z-tests were conducted to detect significant differences between proportions of categories of the variables describing inadequate prescription of VKAs and DOACs; significance was concluded for values of $p<0.05$.

\section{Results}

The SIDIAP database contained 97,350 adult patients with active AF identified in 2018 (Figure 1). Of these, 60,978 were patients with NVAF who were receiving anticoagulant therapy and who fulfilled the inclusion criteria. The mean age was 78 years (SD $=9$ years) and $50.7 \%$ were men. The sociodemographic characteristics, cardiovascular risk factors and morbidities of the study patients, including percentages with missing data, are summarized in Table 4.

Table 4. Sociodemographic study population characteristics, cardiovascular risks factors and comorbidities.

\begin{tabular}{|c|c|c|}
\hline & Total & $\%$ \\
\hline Total & 60,978 & \\
\hline \multicolumn{3}{|l|}{ Gender } \\
\hline Female & 30,085 & 49.3 \\
\hline Male & 30,893 & 50.7 \\
\hline Age (years, mean, SD) & $78(9)$ & \\
\hline \multicolumn{3}{|l|}{ Age (year range) } \\
\hline$<60$ & 1966 & 3.2 \\
\hline $60-69$ & 6158 & 10.1 \\
\hline 70-79 & 18,794 & 30.8 \\
\hline$\geq 80$ & 34,060 & 55.9 \\
\hline \multicolumn{3}{|l|}{ First OA prescribed } \\
\hline VKA & 52,914 & 86.8 \\
\hline DOAC & 7974 & 13.1 \\
\hline OA prescription in $\mathrm{PC}$ & 42,272 & 69.3 \\
\hline \multicolumn{3}{|l|}{ Cardiovascular history } \\
\hline Peripheral arteriopathy & 4193 & 6.9 \\
\hline Ischemic cardiopathy & 11,622 & 19.1 \\
\hline Aortic atherosclerosis & 634 & 1.0 \\
\hline Ischemic stroke or TIA & 11,659 & 19.1 \\
\hline Intracranial hemorrhage & 826 & 1.4 \\
\hline \multicolumn{3}{|l|}{ Morbidity } \\
\hline Arterial hypertension & 48,547 & 79.6 \\
\hline Heart failure & 16,099 & 26.4 \\
\hline Kidney failure & 17,621 & 28.9 \\
\hline \multicolumn{3}{|l|}{ History of bleeding risk } \\
\hline Alcohol & 2570 & 4.2 \\
\hline
\end{tabular}


Table 4. Cont.

\begin{tabular}{|c|c|c|}
\hline & Total & $\%$ \\
\hline Intracranial aneurysm & 63 & 0.1 \\
\hline Portal hypertension & 104 & 0.2 \\
\hline Liver failure & 381 & 0.6 \\
\hline Hereditary telangiectasia & 4 & 0.0 \\
\hline Active aneurysm and dissection of aorta & 936 & 1.5 \\
\hline Gastrointestinal angiodysplasia & 177 & 0.3 \\
\hline Hemorrhages other than digestive and intracranial & 838 & 1.4 \\
\hline $\begin{array}{c}\text { Gastrointestinal hemorrhage } \\
\text { Scores } \\
\mathrm{CHA}_{2} \mathrm{DS}_{2} \mathrm{VASc}\end{array}$ & 5138 & 8.4 \\
\hline 0 & 903 & 1.5 \\
\hline 1 & 3720 & 6.1 \\
\hline 2 & 10,723 & 17.6 \\
\hline 3 & 20,501 & 33.6 \\
\hline$\geq 4$ & 25,131 & 41.2 \\
\hline \multicolumn{3}{|l|}{ HAS-BLED } \\
\hline 0 & 2016 & 3.3 \\
\hline 1 & 22,145 & 36.3 \\
\hline 2 & 21,640 & 35.5 \\
\hline 3 & 10,947 & 18.0 \\
\hline$\geq 4$ & 4230 & 6.9 \\
\hline \multicolumn{3}{|l|}{$\mathrm{e}$ GFR $\mathrm{mL} / \mathrm{min} / 1.73 \mathrm{~m}^{2}$} \\
\hline$<15$ & 384 & 0.6 \\
\hline $15-29$ & 2839 & 4.7 \\
\hline $30-49$ & 12,212 & 20.0 \\
\hline$\geq 50$ & 39,447 & 64.7 \\
\hline Missing & 6096 & 10.0 \\
\hline \multicolumn{3}{|l|}{ Patients visited outside PPC } \\
\hline Domiciliary care & 7832 & 12.8 \\
\hline Institutionalized & 2662 & 4.4 \\
\hline
\end{tabular}

VKA: vitamin K antagonist; DOAC: direct oral anticoagulant; OA: oral anticoagulant; PC: primary care, TIA: transient ischemic attack; PPC: primary care centre; eGFR: glomerular filtration estimated by CKD-EPI $\left(\mathrm{mL} / \mathrm{min} / 1.73 \mathrm{~m}^{2}\right)$.

The main study variable is OA adequacy. OA adequacy characteristics, based on the official adequacy criteria [3,4], are described.

Regarding OA therapy, 41,430 (68\%) had VKA prescription and 19,548 (32\%) had DOAC prescription (Table 5).

The main outcome variable was OA adequacy, based on the following official adequacy criteria $[3,4]$ : 
Table 5. Inadequate prescribing of VKAs and DOACs by patient characteristics.

\begin{tabular}{|c|c|c|c|c|c|c|c|c|}
\hline & \multicolumn{4}{|c|}{ Inadequate Prescribing of VKAs } & \multicolumn{4}{|c|}{ Inadequate Prescribing of DOACs } \\
\hline & Total & $\mathbf{n}$ & $\%$ & ${ }^{a} p$ & Total & $\mathbf{n}$ & $\%$ & ${ }^{a} p$ \\
\hline Total & 41,430 & 14,909 & 36.0 & & 19,548 & 13,218 & 67.6 & \\
\hline \multicolumn{9}{|l|}{ Gender } \\
\hline Female & 20,285 & 7671 & 37.8 & \multirow{2}{*}{$<0.001$} & 9800 & 6550 & 66.8 & \multirow{2}{*}{0.019} \\
\hline Male & 21,145 & 7238 & 34.2 & & 9748 & 6668 & 68.4 & \\
\hline \multicolumn{9}{|l|}{ Age } \\
\hline$<60^{b}$ & 935 & 347 & 37.1 & & 1031 & 851 & 82.5 & \\
\hline $60-69$ & 3774 & 1303 & 34.5 & 0.138 & 2384 & 1808 & 75.8 & $<0.001$ \\
\hline $70-79$ & 12,893 & 4400 & 34.1 & 0.063 & 5901 & 4090 & 69.3 & $<0.001$ \\
\hline$\geq 80$ & 23,828 & 8859 & 37.2 & 0.967 & 10,232 & 6469 & 63.2 & $<0.001$ \\
\hline \multicolumn{9}{|l|}{ First OA prescribed } \\
\hline VKA & 40,671 & 14,568 & 35.8 & \multirow[b]{2}{*}{0.385} & 12,243 & 6425 & 52.5 & \multirow[b]{2}{*}{$<0.001$} \\
\hline DOAC & 669 & 251 & 37.5 & & 7305 & 6793 & 93.0 & \\
\hline \multicolumn{9}{|l|}{ OA prescription in $\mathrm{PC}$} \\
\hline Yes & 33,263 & 11,861 & 35.7 & \multirow{2}{*}{0.005} & 9009 & 6568 & 72.9 & \multirow{2}{*}{$<0.001$} \\
\hline No & 8167 & 3048 & 37.3 & & 10,539 & 6650 & 63.1 & \\
\hline \multicolumn{9}{|l|}{ Cardiovascular history } \\
\hline Peripheral arteriopathy & 2704 & 1090 & 40.3 & \multirow{2}{*}{$<0.001$} & 1489 & 710 & 47.7 & \multirow{2}{*}{$<0.001$} \\
\hline No & 38,726 & 13,819 & 35.7 & & 18,059 & 12,508 & 69.3 & \\
\hline Ischemic cardiomyopathy & 7514 & 2876 & 38.3 & \multirow{2}{*}{$<0.001$} & 4108 & 2269 & 55.2 & \multirow[b]{2}{*}{$<0.001$} \\
\hline No & 33,916 & 12,033 & 35.5 & & 15,440 & 10,949 & 70.9 & \\
\hline Aortic atherosclerosis & 400 & 144 & 36.0 & \multirow{2}{*}{0.995} & 234 & 119 & 50.9 & \multirow{2}{*}{$<0.001$} \\
\hline No & 41,030 & 14,765 & 36.0 & & 19,314 & 13,099 & 67.8 & \\
\hline Ischemic stroke or TIA & 6768 & 2576 & 38.1 & \multirow[b]{2}{*}{$<0.001$} & 4891 & 2025 & 41.4 & \multirow[b]{2}{*}{$<0.001$} \\
\hline No & 34,662 & 12,333 & 35.6 & & 14,657 & 11,193 & 76.4 & \\
\hline Intracranial hemorrhage & 364 & 329 & 90.4 & & 462 & 58 & 12.6 & \\
\hline No & 41,066 & 14,580 & 35.5 & $<0.001$ & 19,086 & 13,160 & 69.0 & $<0.001$ \\
\hline Morbidity & & & & & & & & \\
\hline Diabetes mellitus & 13,587 & 5412 & 39.8 & & 6474 & 3939 & 60.8 & \\
\hline No & 27,843 & 9497 & 34.1 & $<0.001$ & 13,074 & 9279 & 71.0 & $<0.001$ \\
\hline Arterial hypertension & 33,360 & 12,085 & 36.2 & & 15,187 & 9934 & 65.4 & \\
\hline No & 8070 & 2824 & 35.0 & 0.038 & 4361 & 3284 & 75.3 & $<0.001$ \\
\hline Heart failure & 10,846 & 4348 & 40.1 & & 5253 & 3232 & 61.5 & \\
\hline No & 30,584 & 10,561 & 34.5 & $<0.001$ & 14,295 & 9986 & 69.9 & $<0.001$ \\
\hline Kidney failure & 12,340 & 4760 & 38.6 & & 5281 & 3117 & 59.0 & \\
\hline No & 29,090 & 10,149 & 34.9 & $<0.001$ & 14,267 & 10,101 & 70.8 & $<0.001$ \\
\hline History of bleeding risk & & & & & & & & \\
\hline Alcohol & 1727 & 722 & 41.8 & & 843 & 518 & 61.4 & \\
\hline No & 39,703 & 14,187 & 35.7 & $<0.001$ & 18,705 & 12,700 & 67.9 & $<0.001$ \\
\hline Intracranial aneurysm & 28 & 12 & 42.9 & & 35 & 8 & 22.9 & \\
\hline No & 41,402 & 14,897 & 36.0 & 0.449 & 19,513 & 13,210 & 67.7 & $<0.001$ \\
\hline Portal hypertension & 80 & 28 & 35.0 & & 24 & 17 & 70.8 & \\
\hline No & 41,350 & 14,881 & 36.0 & 0.854 & 19,524 & 13,201 & 67.6 & 0.736 \\
\hline Liver failure & 263 & 111 & 42.2 & & 118 & 80 & 67.8 & \\
\hline No & 41,167 & 14,798 & 35.9 & 0.035 & 19,430 & 13,138 & 67.6 & 0.967 \\
\hline Hereditary telangiectasia & 2 & 1 & 50.0 & & & 2 & 100.0 & \\
\hline No & 41,428 & 14,908 & 36.0 & 0.680 & 19,546 & 13,216 & 67.6 & 0.328 \\
\hline
\end{tabular}


Table 5. Cont.

\begin{tabular}{|c|c|c|c|c|c|c|c|c|}
\hline & \multicolumn{4}{|c|}{ Inadequate Prescribing of VKAs } & \multicolumn{4}{|c|}{ Inadequate Prescribing of DOACs } \\
\hline & Total & n & $\%$ & ${ }^{\mathbf{a}} \mathbf{p}$ & Total & n & $\%$ & ${ }^{a} p$ \\
\hline \multirow{2}{*}{$\begin{array}{l}\text { Active aneurysm and } \\
\text { dissection of aorta } \\
\text { No }\end{array}$} & 614 & 219 & 35.7 & \multirow[t]{2}{*}{0.869} & 322 & 202 & 62.7 & \multirow[t]{2}{*}{0.059} \\
\hline & 40,816 & 14,690 & 36.0 & & 19,226 & 13,016 & 67.7 & \\
\hline Gastrointestinal angiodysplasia & 92 & 41 & 44.6 & \multirow{2}{*}{0.086} & 85 & 57 & 67.1 & \multirow{2}{*}{0.912} \\
\hline No & 41,338 & 14,868 & 36.0 & & 19,463 & 13,161 & 67.6 & \\
\hline Hemorrhages other than digestive & 569 & 202 & 35.5 & \multirow{2}{*}{0.808} & 269 & 165 & 61.3 & \multirow{2}{*}{0.027} \\
\hline No & 40,861 & 14,707 & 36.0 & & 19,279 & 13,053 & 67.7 & \\
\hline Gastrointestinal hemorrhage & 3286 & 1280 & 39.0 & \multirow{2}{*}{$<0.001$} & 1852 & 1150 & 62.1 & \multirow{2}{*}{$<0.001$} \\
\hline No & 38,144 & 13,629 & 35.7 & & 17,696 & 12,068 & 68.2 & \\
\hline \multicolumn{9}{|l|}{ Scores } \\
\hline \multicolumn{9}{|l|}{$\mathrm{CHA}_{2} \mathrm{DS}_{2}$ VASc } \\
\hline $0^{\mathrm{b}}$ & 404 & 127 & 31.4 & & 499 & 450 & 90.2 & \\
\hline 1 & 2107 & 661 & 31.4 & 0.980 & 1613 & 1365 & 84.6 & 0.002 \\
\hline 2 & 7251 & 2321 & 32.0 & 0.810 & 3472 & 2695 & 77.6 & $<0.001$ \\
\hline 3 & 14,681 & 5138 & 35.0 & 0.138 & 5820 & 4092 & 70.3 & $<0.001$ \\
\hline$\geq 4$ & 16,987 & 6662 & 39.2 & 0.002 & 8144 & 4616 & 56.7 & $<0.001$ \\
\hline \multicolumn{9}{|l|}{ HAS-BLED } \\
\hline $0^{\mathrm{b}}$ & 953 & 156 & 16.4 & & 1063 & 1003 & 94.4 & \\
\hline 1 & 14,563 & 2343 & 16.1 & 0.819 & 7582 & 6478 & 85.4 & $<0.001$ \\
\hline 2 & 15,112 & 6158 & 40.7 & $<0.001$ & 6528 & 4243 & 65.0 & $<0.001$ \\
\hline 3 & 7783 & 4308 & 55.4 & $<0.001$ & 3164 & 1181 & 37.3 & $<0.001$ \\
\hline$\geq 4$ & 3019 & 1944 & 64.4 & $<0.001$ & 1211 & 313 & 25.8 & $<0.001$ \\
\hline \multicolumn{9}{|l|}{$\mathrm{e}$ GFR $\mathrm{mL} / \mathrm{min} / 1.73 \mathrm{~m}^{2}$} \\
\hline$<15^{\mathrm{c}}$ & 313 & & & \multirow{5}{*}{$\begin{array}{l}<0.001 \\
<0.001\end{array}$} & 71 & 71 & 100.0 & \multirow{5}{*}{$\begin{array}{l}<0.001 \\
<0.001 \\
<0.001\end{array}$} \\
\hline $15-29^{d}$ & 1850 & 920 & 49.7 & & 989 & 485 & 49.0 & \\
\hline $30-49$ & 8239 & 3616 & 43.9 & & 3973 & 2159 & 54.3 & \\
\hline$\geq 50$ & 27,855 & 10,373 & 37.2 & & 11,592 & 7580 & 65.4 & \\
\hline Missing & 3173 & & & & 2923 & 2923 & 100.0 & \\
\hline \multicolumn{9}{|l|}{ Patients visited outside PPC } \\
\hline Domiciliary care & 4926 & 2134 & 43.3 & \multirow{2}{*}{$<0.001$} & 2906 & 1696 & 58.4 & \multirow{2}{*}{$<0.001$} \\
\hline No & 36,504 & 12,775 & 35.0 & & 16,642 & 11,522 & 69.2 & \\
\hline Institutionalized & 1646 & 739 & 44.9 & \multirow{2}{*}{$<0.001$} & 1016 & 602 & 59.3 & \multirow{2}{*}{$<0.001$} \\
\hline No & 39,784 & 14,170 & 35.6 & & 18,532 & 12,616 & 68.1 & \\
\hline
\end{tabular}

VKAs: vitamin K antagonists; DOACs: direct oral anticoagulants; OA: oral anticoagulant; PC: primary care; TIA: transient ischemic attack; PPC: primary care centre; ${ }_{e}$ GFR: glomerular filtration estimated by CKD-EPI $\left(\mathrm{mL} / \mathrm{min} / 1.73 \mathrm{~m}^{2}\right)$. ${ }^{a}$ Two proportion Z-test. ${ }^{b}$ Reference in VKAs and DOACs. ${ }^{c}$ Reference for DOACs only, ${ }^{d}$ Reference for VKAs only.

\subsection{Adequacy and Inadequacy to DOAC Prescription}

Inadequacy of DOAC prescription was $67.6 \%$, versus $32.4 \%$ of adequacy (Table 5). Inadequate prescription was significantly higher in men (68.4\%) than in women (66.8\%). Up to $82 \%$ of people $<60$ years of age who were treated with DOACs did not meet prescription criteria. Moreover, inadequate DOAC prescription inversely associated with age (Table 5). Up to $69-76.4 \%$ patients with no history of CVD, ICH, morbidity or with an established history of HRB failed to meet the adequacy criteria for DOACs. Inadequate prescription increased inversely with the $\mathrm{CHA}_{2} \mathrm{DS}_{2} \mathrm{VASc}$ and HAS-BLED scores, a value of zero being that most commonly associated with inadequate prescription (Table 5).

Most patients $(77.3 \%)$ had a eGFR $\geq 15$, although this did not fulfill the adequacy criteria for other reasons, for example, because the DOAC was prescribed as the first-line anticoagulant without histories of post-VKA TEE, SHRB or ICH, or of ADR to DOAC 
(Table 6). This treatment is inadequate for patients with $\mathrm{e}$ GFR $<15$ or with a missing GF value $(22.1 \%$ ) (Table 6).

Table 6. Adequate prescribing of direct oral anticoagulants (DOACs) based in the 2016 to the IPT criteria.

\begin{tabular}{|c|c|c|c|c|}
\hline & \multicolumn{2}{|c|}{ Adequate Prescribing of DOACs } & \multicolumn{2}{|c|}{ Inadequate Prescribing of DOACs } \\
\hline & $\mathbf{n}$ & $\%$ & $+\mathbf{n}$ & $\%$ \\
\hline Total & 6330 & 32.4 & 13,218 & 67.6 \\
\hline \multicolumn{5}{|l|}{ eGFR } \\
\hline$<15$ & & & 71 & 0.5 \\
\hline$\geq 15$ & 6330 & 100.0 & 10,224 & $77.3^{\mathrm{a}}$ \\
\hline Missing & & & 2923 & 22.1 \\
\hline ADR DOAC & & & 55 & 0.4 \\
\hline ADR VKA & 45 & 0.7 & 54 & 0.4 \\
\hline \multicolumn{5}{|l|}{ Pre DOAC TTR } \\
\hline$<65 \%$ & 3699 & 58.4 & 276 & 2.1 \\
\hline \multicolumn{5}{|l|}{ GFR } \\
\hline$<15$ & & & 16 & 5.8 \\
\hline$\geq 15$ & & & $9^{b}$ & 3.3 \\
\hline Missing & & & 251 & 90.9 \\
\hline$\geq 65 \%$ & 680 & 10.7 & 1342 & 10.2 \\
\hline Missing & 1439 & 22.7 & 4807 & 36.4 \\
\hline \multicolumn{5}{|l|}{$\begin{array}{c}\text { Cardiovascular } \\
\text { event }\end{array}$} \\
\hline Post VKA TEE & 2589 & 40.9 & 288 & 2.2 \\
\hline \multicolumn{5}{|l|}{ eGFR } \\
\hline$<15$ & & & 13 & 4.5 \\
\hline$\geq 15$ & & & $9^{b}$ & 3.1 \\
\hline Missing & & & 266 & 92.4 \\
\hline ICH & 404 & 6.4 & 58 & 0.4 \\
\hline \multicolumn{5}{|l|}{ eGFR } \\
\hline$<15$ & & & 2 & 3.4 \\
\hline$\geq 15$ & & & $2^{b}$ & 3.4 \\
\hline Missing & & & 54 & 93.1 \\
\hline SHRB & 1754 & 27.7 & 80 & 0.6 \\
\hline \multicolumn{5}{|l|}{ eGFR } \\
\hline$<15$ & & & 17 & 21.3 \\
\hline$\geq 15$ & & & $3^{b}$ & 3.8 \\
\hline Missing & & & 60 & 75.0 \\
\hline
\end{tabular}

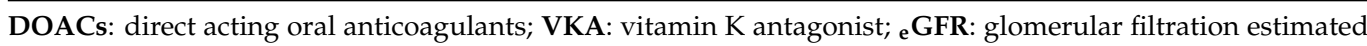
by CKD-EPI $\left(\mathrm{mL} / \mathrm{min} / 1.73 \mathrm{~m}^{2}\right)$; ADR: adverse drug reaction; TTR: time in therapeutic range according to the Rosendaal method; TEE: thromboembolic event; ICH: intracranial hemorrhage; SHRB: stroke with high risk of bleeding; a ADR to DOACs, or DOAC prescribed as first-line anticoagulant without history of post-VKA TEE, $\mathrm{SHRB}$ or $\mathrm{ICH}^{\mathrm{b}}{ }^{\mathrm{b}}$ also have ADR to DOACs.

DOAC prescription was $32.4 \%$ adequate (Table 6). Adequate prescription was concluded for all patients presenting ${ }_{e}$ GFR $\geq 15$ and $58.4 \%$ patients with a pre-DOAC TTR ${ }^{R}$ of $<65 \%$ (who were prescribed a VKA as first-line treatment). In total, $40.9 \%, 27.7 \%$ and $6.4 \%$ of the adequate patients had post-VKA TEE, SHRB and ICH, respectively (see detail in Supplementary Material).

\subsection{Adequacy and Inadequacy to VKA}

The adequacy and inadequacy of the VKA prescription were $64 \%$ and $36 \%$, respectively (Table 5). Inadequate prescription was significantly higher in women (37\%) than in men (34.2\%) (Table 5). Adequacy criteria for VKA therapy were not met in $36.2-41.8 \%$ 
patients treated with VKAs with a history of CVD (except for aortic atherosclerosis), any of the studied morbidities or an established history of HRB. Inadequate prescription was also associated with $\mathrm{ICH}$, and $90.4 \%$ patients with $\mathrm{ICH}$ were inadequately prescribed this treatment (Table 5). Inadequate prescription increased with HAS-BLED scores, reaching $64.4 \%$ when HAS-BLED was $\geq 4$. Patients with $\mathrm{CHA}_{2} \mathrm{DS}_{2} \mathrm{VASc} \geq 4$ had significantly higher rates of inadequacy than those with lower $\mathrm{CHA}_{2} \mathrm{DS}_{2}$ VASc values. Institutionalized patients and those in domiciliary care had significantly higher inadequacy rates (43.3-44.9\%) than did patients who were able to attend the PPC.

Most of the VKA inadequacy ( $98.8 \%$ of cases) was related to VKA prescription when $\mathrm{TTR}^{\mathrm{R}}$ was $<65 \%$ after 6 months of treatment (Table 7 ).

Table 7. Adequate prescribing of vitamin K antagonists (VKAs) based in the 2016 to the IPT criteria.

\begin{tabular}{|c|c|c|c|c|}
\hline & \multicolumn{2}{|c|}{ Adequate Prescribing of VKAs } & \multicolumn{2}{|c|}{ Inadequate Prescribing of VKAs } \\
\hline & $\mathrm{n}$ & $\%$ & $\mathrm{n}$ & $\%$ \\
\hline Total & 26,521 & 64.0 & 14,909 & 36.0 \\
\hline \multicolumn{5}{|l|}{ TTR $^{\mathrm{R}}$} \\
\hline$<65 \%$ & 1379 & 5.2 & 14,732 & 98.8 \\
\hline \multicolumn{5}{|l|}{ eGFR } \\
\hline$<15$ & 196 & 14.2 & & \\
\hline$\geq 15$ & $1^{b}$ & 0.1 & & \\
\hline Missing & 1182 & 85.7 & & \\
\hline$\geq 65 \%$ & 25,140 & 94.8 & 177 & 1.2 \\
\hline \multirow[t]{2}{*}{ ICH } & & & 170 & \\
\hline & & & $7^{\mathrm{a}}$ & \\
\hline Missing & $2^{c}$ & 0.0 & & \\
\hline \multicolumn{5}{|l|}{${ }_{\mathrm{e}}^{\mathrm{GFR}}$} \\
\hline$<15$ & 313 & 1.2 & & \\
\hline$\geq 15$ & 23,035 & 86.9 & 14,909 & 100 \\
\hline Missing & 3173 & 12.0 & & \\
\hline \multicolumn{5}{|l|}{ ADR } \\
\hline VKA & & & 13 & 0.1 \\
\hline DOAC & 6 & 0.0 & $10^{\mathrm{a}}$ & 0.1 \\
\hline ICH & 35 & 0.1 & 329 & 2.2 \\
\hline \multicolumn{5}{|l|}{ eGFR } \\
\hline$<15$ & 8 & 22.9 & & \\
\hline Missing & 27 & 77.1 & & \\
\hline
\end{tabular}

VKAs: vitamin K antagonists; DOACs: direct oral anticoagulants; $\mathbf{T T R}^{\mathbf{R}}$ : time in therapeutic range according to the Rosendaal method; $\mathrm{e}$ GFR: glomerular filtration estimated according to CKD-EPI $\left(\mathrm{mL} / \mathrm{min} / 1.73 \mathrm{~m}^{2}\right)$; ICH: intracranial hemorrhage; ADR: adverse drug reaction; ${ }^{a}$ ADR VKA. ${ }^{b}$ ADR DOAC. ${ }^{c}$ Insufficient INR to calculate $\mathrm{TTR}^{\mathrm{R}}$.

Adequacy criteria for VKAs were met in $64 \%$ of cases (Table 7). Patients with $\mathrm{TTR}^{\mathrm{R}}<65 \%$ were adequately prescribed a VKA because of a missing ${ }_{\mathrm{e}}$ GFR value, $\mathrm{e}$ GFR $<15$ or ADR to DOACs. Most were adequately prescribed with respect to $\mathrm{TTR}^{\mathrm{R}} \geq 65 \%(94.8 \%$ ), but 170 had ICH and seven presented ADR to VKAs.

\section{Discussion}

This population-based study demonstrates the adequacy of anticoagulant treatment according to IPT [4] criteria in PC patients with NVAF in 2018 in Catalonia. To our knowledge, this is the first real-word data study with a large cohort of Catalan population that evaluates prescription adequacy of anticoagulant therapy for patients with NVAF in relation to IPT [4] criteria (also incorporated into the PHT [3]). Inadequate treatment was more common for DOACs (67.6\%) than for VKAs (36\%). Most of the inadequacy of DOAC prescription arose because it was prescribed as first-line anticoagulant when there was no history of TEEs or ICH. Another substantial part of the inadequacy stems from missing 
eGFR values. Common causes of inadequate VKA prescription were poor TTR control and some cases of ICH.

The European Society of Cardiology [2] and the American Heart Association [1] were prompted to recommend the use of DOACs as first-line treatment instead of VKAs, because the results of pivotal clinical trials with DOACs [5-8,19] showed a reduction in the incidence of stroke by at least the same degree as produced by warfarin, and presented a lower rate of $\mathrm{ICH}$.

More recently, some real-world effectiveness studies have highlighted the limitations of the pivotal DOAC clinical trials, showing that DOACs and warfarin have similar effectiveness and safety with respect to TTE and severe non-intracranial hemorrhages [20,21]. Some studies also showed similar bleeding ratios for DOACs and VKAs [22]. Conversely, other studies with real-world data that compared warfarin and DOACs underlined the effectiveness of DOACs for preventing TTE and reducing the risk of intracranial hemorrhage [23,24]. To resolve these conflicting results, health institutions require costeffectiveness studies based on real-world data that analyze DOACs and VKAs in their specific settings. Recent studies have shown that DOACs are more cost-effective for patients who are poorly controlled with VKAs and for patients at high risk of thromboembolism and bleeding $[25,26]$, supporting the IPT's recommendations concerning DOAC use in specific situations.

In our study, control was poor (TTR $<65 \%$ ) in $38.8 \%$ patients who received VKAs, similar to the levels in other regions of Spain (39.4\%) [27]. TTR was calculated based on the INR and the timings registered in the EHRs. Poor control of anticoagulation is associated with increased risks of stroke, bleeding and all-cause mortality $[28,29]$. Therefore, good control of the INR is essential for the patient, but often not achieved in Catalonia [27], Spain or other countries [30]. Typically, patients treated with VKAs are infracoagulated and not so much overcoagulated, thus increasing the risk of TTE and not so much the bleeding risk. Management of VKAs outside of clinical trials is associated with poor control, particularly at the start of VKA therapy. Good access to TTR values in PC would improve the control of patients treated with VKAs and decision-making regarding adequate switching to DOACs [31], although most PCCs in Spain do not currently have access to such information. On the other hand, patients who receive VKAs and have had HIC should be considered for switching to DOAC as this has been shown to be superior in preventing ICH.

Measurement of GFR is essential for choosing anticoagulant therapy. The absence of this information leads to inadequate prescribing of DOAC. In clinical practice, various equations estimate the GFR [32], one of the most commonly used for DOACs being the CKD-EPI [18]. This was developed to improve the estimate, and is the one we use in our own setting. Not all PCCs include this calculated formula in their EHRs, which hinders the determination of the correct DOAC dose and the transition between VKAs and DOACs. Importantly, while VKAs can be used regardless of the GFR value, it can determine the adequacy in the case of DOACs [4]. For instance, they are contraindicated in some cases. Dabigatran cannot be used with eGFR $<30 \mathrm{~mL} / \mathrm{min}$, and rivaroxaban, apixaban and edoxaban when ${ }_{\mathrm{e}} \mathrm{GFR}$ is $<15$ [4]. In this study it was not differentiated the DOAC type and therefore eGFR $<15$ was considered to be the contraindication criterion of most DOAC. PCCs can access hospital creatinine results, and the number of laboratories providing GFR calculations to adjust anticoagulants is increasing. In this study, we used creatinine to estimate GFR. Records of a substantial proportion of patients receiving DOACs lack a measurement of creatinine from the previous year. It was not possible to estimate their eGFR, which is essential for deciding the prescription and for adjusting the DOAC treatment dose. Probably, the lack of experience on DOACs management, in our setting, explains the absence of ${ }_{e}$ GFR in patients receiving this treatment. This study wants to provide evidence for this problem. While VKA treatments can be adjusted without recourse to renal function information, this is not possible for DOACs. The increase and decrease of DOAC plasma levels, which are closely related to renal function, are associated with 
hemorrhagic and ischemic events, respectively [33]. Thus, eGFR is essential to initiate dosing and to monitor the appropriate dose of DOAC, avoiding problems with DOACs underdoses and overdoses. DOAC dose adjustment is based primarily on $\mathrm{e} G F R$, and other factors such as age, weight and interactions with other drugs.

A report from our setting concerning the use of anticoagulants in NVAF, based on 2014-2017 data, showed the same usage of VKAs $(69 \%)$ and DOACs (31\%) as in our current study (VKAs, 68\%; DOACs, 32\%) [34]. The same study also showed that $29.5 \%$ of patients treated with DOACs lacked clinical data with which to evaluate the dosage correctly [34]. Measures are currently being evaluated and used to increase the availability of GFR determinations in PCCs of the ICS for patients treated with DOACs. To ensure that dosage determination takes kidney function into account, each physician can check their patients who are treated with a DOAC, with no measurement of eGFR recorded during the previous 12 months.

A history of ICH should be considered when prescribing DOACs [4], and treatment should be personalized. Clinical trials have shown the effectiveness of DOACs compared with warfarin at reducing stroke and mortality. DOACs involve a similar risk of major bleeding as with warfarin, but a lower risk of ICH and a higher risk of intestinal bleeding [35].

Results of clinical trials are often difficult to translate into clinical practice, since patients often have more comorbidities, take more medication and cannot be followed up so closely. With regard to ICH, effectiveness studies with real-world data have confirmed that DOACs have fewer associated risks than VKAs [23], so the IPT advises DOAC treatment in such cases [4]. Most patients with a history of ICH received DOACs, probably because they were started on them after this event.

Studies also reveal the risk of gastrointestinal hemorrhage with DOACs $[5,19]$. A history of gastrointestinal hemorrhage is more common in patients treated with DOACs than with VKAs. Even though gastrointestinal hemorrhage is not a criterion in the IPT, clinicians need to consider this risk and advise their patients accordingly.

This study provides a real-world snapshot of anticoagulation in patients with NVAF. We consider that these recently acquired data on adequacy with respect to the criteria in force in Catalonia and Spain could be generalized to other geographical areas. Our results highlight the urgency of facilitating access to TTR by all specialists who manage anticoagulants, and the need for renal function measurements when DOACs are prescribed. It also shows that DOACs are frequently prescribed to young patients who have not previously experienced either the TEE or ICH that would justify this choice.

The study has some limitations. First, the cross-sectional design, used to investigate current patterns, does not allow causal inferences to be made. Nevertheless, it serves to generate hypotheses that could be examined in further studies. Second, there was a high frequency of missing scores, which had to be calculated from available patient data. Type of reporting and underreporting might constitute a limitation in this real-word data based study. The IPT does not take into account treatment adherence or lack of access to INR monitoring. However, access to INR is very high in PC patients of the ICS, considering that patients who are unable to attend the PCC are monitored at home. Guidelines agreed by experts and based on scientific evidence are key instruments for making individual decisions, although other factors, such as compliance and adherence to drugs, must frequently be considered. Both, compliance with VKA and adherence to DOAC are key factors in oral anticoagulant treatment in NVAF and should be consider in further studies.

The Spanish IPT criteria are more restrictive with respect to the use of DOACs than the European guidelines, although both are supported by scientific evidence. Regarding the main factors associated with inadequate OA prescription, our study highlights the need for improved strategies that can be generalized to other locations. The OA prescription criteria of different countries' guidelines can differ in aspects related to first-line treatment 
or specific situations when the use of DOACs or VKAs is recommended. However, in clinical practice, the adherence to official criteria guidelines improves patient safety.

\section{Conclusions}

In patients with NVAF, prescription adequacy was higher for VKAs than for DOACs. Most of the inadequacy of DOACs arises from its prescription as first-line anticoagulant in patients without a history of TEEs or of ICH. A substantial proportion of the inadequacy is also explained by missing eGFR values. Common causes of inadequate VKA prescription were poor TTR control and some cases of ICH.

TTR and eGFR should be routinely calculated and recorded in the EHRs to facilitate decision-making. Main factors associated with inadequate OA prescription and the improved strategies identified in the present study are useful for improving clinical practice and patient safety in the health systems of different countries.

Supplementary Materials: The following are available online at https:/ /www.mdpi.com/1660-460 1/18/5/2244/s1, Figure S1: Detail of inadequate DOAC prescription.

Author Contributions: M.R.D.L., C.A.M., A.Q.G. conceived and designed the study. M.R.D.L., N.C.-Q. wrote the manuscript. M.R.D.L., E.C.B. and J.F.S. are guarantors and take full responsibility for the integrity of the data and the accuracy of the data analysis. M.R.D.L., C.A.M., A.Q.G., J.M.P.V., D.R.C. and E.F.D. participated in the study design and methodology. M.R.D.L., N.C.-Q. and Z.H.R. reviewed and update the bibliography. J.F.-S. performed the analysis. All authors participated in interpretation of data and contributed to the critical revision of the manuscript for important intellectual content. All authors have read and agreed to the published version of the manuscript.

Funding: Department of Health of the Generalitat de Catalunya, Strategic Plan for Research and Innovation in Health (PERIS). Research project number SLT 002/16/00146 and Intensification of Specialist Physicians SLT008/18/00021 grant.

Institutional Review Board Statement: The study was conducted according to the guidelines of the Declaration of Helsinki, and approved by the Institutional Review Board (or Ethics Committee) of IDIAP Jordi Gol (protocol code P17/091, 15th March 2017).

Informed Consent Statement: Not applicable.

Data Availability Statement: The data that support the findings of this study were obtained from SIDIAP database (Information System for Research in Primary Care). This database is representative of the Catalan population. Restrictions apply to the availability of these data, which were used under license for this study. The authors have no authorization to share the data.

Acknowledgments: The authors thank the following Departments for their contribution: Primary Care Management of the Catalan Institute of Health, Information Systems of the Primary Care Services, Regional Management and Primary Care Management of the Terres de l'Ebre, Unit of Information Systems of the Regional Management Terres de l'Ebre, Functional Competences Centre of the ECAP of the Information Systems Area and SIDIAP. M.R.D.L. thanks the Department of Health of the Generalitat de Catalunya for her Intensification of Specialist Physicians SLT008/18/00021 PERIS grant. This study obtained competitive, public funding from the Health Department, as part of the Health Research and Innovation Strategic Plan (PERIS) 2016-2020, number SLT 002/16/00146. This project was carried out with the support of the Department of Health of the Generalitat de Catalunya, in the 2019 round of grants for the Strategic Plan for Research and Innovation in Health (PERIS) 2016-2020.

Conflicts of Interest: M.R.D.L. declares having received funding to attend Bayer conferences, and partial sponsorship of a study funded by Bayer. C.A.M., A.Q.G., D.R.C., J.M.P.V., and J.F.-S. declare having received partial sponsorship of a study funded by Bayer. Z.H.R. declares having received funding to attend and participate in conferences Almirall and Boehringer, and partial sponsorship of a study funded by Bayer. E.F.D. declares having received funding to attend meetings sponsored by MSD and GlaxoSmithKline, and having received partial sponsorship of a study funded by Bayer. None of the sponsors were involved in the design and conduct of the study; the collection, management, analysis, and interpretation of the data; or the preparation, review or approval of the manuscript, or the decision to submit it for publication. 


\section{References}

1. January, C.T.; Wann, L.S.; Calkins, H.; Chen, L.Y.; Cigarroa, J.E.; Cleveland, J.C., Jr.; Ellinor, P.T.; Ezekowitz, M.D.; Field, M.E.; Furie, K.L.; et al. AHA/ACC/HRS Focused Update of the 2014 AHA/ACC/HRS Guideline for the Management of Patients With Atrial Fibrillation: A Report of the American College of Cardiology/American Heart Association Task Force on Clinical Practice Guidelines and the Heart R. Circulation 2019, 140, e125-e151. [CrossRef] [PubMed]

2. Kirchhof, P.; Benussi, S.; Kotecha, D.; Ahlsson, A.; Atar, D.; Casadei, B.; Castella, M.; Diener, H.C.; Heidbuchel, H.; Hendriks, J.; et al. 2016 ESC Guidelines for the management of atrial fibrillation developed in collaboration with EACTS. Eur. Hear. J. 2016, 37, 2893-2962. [CrossRef]

3. Programa d'harmonització farmacoterapèutica. Pautes per a L'harmonització de l'ús d'aNticoagulants Orals per a la Prevenció de l'ictus i l'embòlia Sistèmica en Pacients amb Fibril-Lació Auricular; Servei Català de la Salut; Departament de Salut; Generalitat de Catalunya: Barcelona, Spain, 2018; pp. 1-76.

4. Ministerio de Sanidad, S.S. Criterios y Recomendaciones Generales para el uso de los Anticoagulantes orales Directos (ACOD) en la Prevención del ictus y la Embolia Sistémica en Pacientes con Fibrilación Auricular no Valvular; Informe de posicionamento terapéutico UT-ACOD/V5/21112016: Madrid, Spain, 2016.

5. Connolly, S.J.; Ezekowitz, M.D.; Yusuf, S.; Eikelboom, J.; Oldgren, J.; Parekh, A.; Pogue, J.; Reilly, P.A.; Themeles, E.; Varrone, J.; et al. Dabigatran versus warfarin in patients with atrial fibrillation. N. Engl. J. Med. 2009, 361, 1139-1151. [CrossRef] [PubMed]

6. Patel, M.R.; Mahaffey, K.W.; Garg, J.; Pan, G.; Singer, D.E.; Hacke, W.; Breithardt, G.; Halperin, J.L.; Hankey, G.J.; Piccini, J.P.; et al. Rivaroxaban versus warfarin in nonvalvular atrial fibrillation. N. Engl. J. Med. 2011, 365, 883-891. [CrossRef] [PubMed]

7. Granger, C.B.; Alexander, J.H.; McMurray, J.J.; Lopes, R.D.; Hylek, E.M.; Hanna, M.; Al-Khalidi, H.R.; Ansell, J.; Atar, D.; Avezum, A.; et al. Apixaban versus warfarin in patients with atrial fibrillation. N. Engl. J. Med. 2011, 365, 981-992. [CrossRef]

8. Giugliano, R.P.; Ruff, C.T.; Braunwald, E.; Murphy, S.A.; Wiviott, S.D.; Halperin, J.L.; Waldo, A.L.; Ezekowitz, M.D.; Weitz, J.I.; Spinar, J.; et al. Edoxaban versus warfarin in patients with atrial fibrillation. N. Engl. J. Med. 2013, 369, $2093-2104$. [CrossRef] [PubMed]

9. Ibáñez, L.; Sabaté, M.; Vidal, X.; Ballarin, E.; Rottenkolber, M.; Schmiedl, S.; Heeke, A.; Huerta, C.; Martin Merino, E.; Montero, D.; et al. Incidence of direct oral anticoagulant use in patients with nonvalvular atrial fibrillation and characteristics of users in 6 European countries (2008-2015): A cross-national drug utilization study. Br. J. Clin. Pharmacol. 2019. [CrossRef]

10. Troncoso, A.; Diogène, E. Dabigatran and rivaroxaban prescription for atrial fibrillation in Catalonia, Spain: The need to manage the introduction of new drugs. Eur. J. Clin. Pharmacol. 2014, 70, 249-250. [CrossRef]

11. Bolíbar, B.; Fina Avilés, F.; Morros, R.; Del Mar Garcia-Gil, M.; Hermosilla, E.; Ramos, R.; Rosell, M.; Rodríguez, J.; Medina, M.; Calero, S.; et al. Base de datos SIDIAP: La historia clínica informatizada de Atención Primaria como fuente de información para la investigación epidemiológica. Med. Clin. 2012, 138, 617-621. [CrossRef]

12. Del Mar García-Gil, M.; Hermosilla, E.; Prieto-Alhambra, D.; Fina, F.; Rosell, M.; Ramos, R.; Rodriguez, J.; Williams, T.; Van Staa, T.; Bolíbar, B. Construction and validation of a scoring system for the selection of high-quality data in a Spanish population primary care database (SIDIAP). Inform. Prim. Care 2012, 19, 135-145. [CrossRef] [PubMed]

13. WHOCC WHOCC_ATC/DDD Index. Available online: https:/ / www.whocc.no/atc_ddd_index/ (accessed on 26 November 2020).

14. WHO (World Health Organization) Classification of Diseases (ICD). Available online: https:/ / www.who.int/classifications / classification-of-diseases (accessed on 26 November 2020).

15. Rosendaal, F.R.; Cannegieter, S.C.; van der Meer, F.J.; Briet, E. A method to determine the optimal intensity of oral anticoagulant therapy. Thromb Haemost 1993, 69, 236-239. [CrossRef]

16. Olesen, J.; Torp-Pedersen, C.; Hansen, M.; Lip, G. The value of the CHA2DS2-VASc score for refining stroke risk stratification in patients with atrial fibrillation with a CHADS2 score 0-1: A nationwide cohort study. Thromb. Haemost. 2012, 107, 1172-1179. [CrossRef]

17. Pisters, R.; Lane, D.A.; Nieuwlaat, R.; de Vos, C.B.; Crijns, H.J.G.M.; Lip, G.Y.H. A Novel User-Friendly Score (HAS-BLED) To Assess 1-Year Risk of Major Bleeding in Patients with Atrial Fibrillation. Chest 2010, 138, 1093-1100. [CrossRef]

18. Levey, A.S.; Stevens, L.A.; Schmid, C.H.; Zhang, Y.L.; Castro, A.F.; Feldman, H.I.; Kusek, J.W.; Eggers, P.; Van Lente, F.; Greene, T.; et al. A new equation to estimate glomerular filtration rate. Ann. Intern. Med. 2009, 150, 604-612. [CrossRef] [PubMed]

19. Schmitt, L.; Speckman, J.; Ansell, J. Quality assessment of anticoagulation dose management: Comparative evaluation of measures of time-in-therapeutic range. J. Thromb. Thrombolysis 2003, 15, 213-216. [CrossRef] [PubMed]

20. Forslund, T.; Wettermark, B.; Andersen, M.; Hjemdahl, P. Stroke and bleeding with non-Vitamin K antagonist oral anticoagulant or warfarin treatment in patients with non-valvular atrial fibrillation: A population-based cohort study. Europace 2018, 20, 420-428. [CrossRef] [PubMed]

21. Laliberté, F.; Cloutier, M.; Nelson, W.W.; Coleman, C.I.; Pilon, D.; Olson, W.H.; Damaraju, C.V.; Schein, J.R.; Lefebvre, P. Real-world comparative effectiveness and safety of rivaroxaban and warfarin in nonvalvular atrial fibrillation patients. Curr. Med. Res. Opin. 2014, 30, 1317-1325. [CrossRef]

22. Ellis, M.H.; Neuman, T.; Bitterman, H.; Dotan, S.G.; Hammerman, A.; Battat, E.; Eikelboom, J.W.; Ginsberg, J.S.; Hirsh, J. Bleeding in patients with atrial fibrillation treated with dabigatran, rivaroxaban or warfarin: A retrospective population-based cohort study. Eur. J. Intern. Med. 2016, 33, 55-59. [CrossRef]

23. Coleman, C.I.; Antz, M.; Ehlken, B.; Evers, T. REal-LIfe Evidence of stroke prevention in patients with atrial Fibrillation-The RELIEF study. Int. J. Cardiol. 2016, 203, 882-884. [CrossRef] 
24. Li, X.S.; Deitelzweig, S.; Keshishian, A.; Hamilton, M.; Horblyuk, R.; Gupta, K.; Luo, X.; Mardekian, J.; Friend, K.; Nadkarni, A.; et al. Effectiveness and safety of apixaban versus warfarin in non-valvular atrial fibrillation patients in "real-world" clinical practice. A propensity-matched analysis of 76,940 patients. Thromb. Haemost. 2017, 117, 1072-1082. [CrossRef]

25. Coyle, D.; Coyle, K.; Cameron, C.; Lee, K.; Kelly, S.; Steiner, S.; Wells, G.A. Cost-effectiveness of new oral anticoagulants compared with warfarin in preventing stroke and other cardiovascular events in patients with atrial fibrillation. Value Health 2013, 16, 498-506. [CrossRef] [PubMed]

26. Amin, A.; Keshishian, A.; Vo, L.; Zhang, Q.; Dina, O.; Patel, C.; Odell, K.; Trocio, J. Real-world comparison of all-cause hospitalizations, hospitalizations due to stroke and major bleeding, and costs for non-valvular atrial fibrillation patients prescribed oral anticoagulants in a US health plan. J. Med. Econ. 2018, 21, 244-253. [CrossRef] [PubMed]

27. Barrios, V.; Escobar, C.; Prieto, L.; Osorio, G.; Polo, J.; Lobos, J.M.; Vargas, D.; García, N. Anticoagulation Control in Patients with Nonvalvular Atrial Fibrillation Attended at Primary Care Centers in Spain: The PAULA Study. Rev. Española Cardiol. 2015, 68, 769-776. [CrossRef]

28. Cancino, R.S.; Hylek, E.M.; Reisman, J.I.; Rose, A.J. Comparing patient-level and site-level anticoagulation control as predictors of adverse events. Thromb. Res. 2014, 133, 652-656. [CrossRef]

29. Gallagher, A.M.; Setakis, E.; Plumb, J.M.; Clemens, A.; van Staa, T.-P. Risks of stroke and mortality associated with suboptimal anticoagulation in atrial fibrillation patients. Thromb. Haemost. 2011, 106, 968-977. [CrossRef]

30. Mearns, E.S.; White, C.M.; Kohn, C.G.; Hawthorne, J.; Song, J.S.; Meng, J.; Schein, J.R.; Raut, M.K.; Coleman, C.I. Quality of vitamin $\mathrm{K}$ antagonist control and outcomes in atrial fibrillation patients: A meta-analysis and meta-regression. Thromb. J. 2014, 12, 14. [CrossRef]

31. Dalmau Llorca, M.R.; Gonçalves, A.Q.; Forcadell Drago, E.; Fernández-Sáez, J.; Hernández Rojas, Z.; Pepió Vilaubí, J.M.; Rodríguez Cumplido, D.; Morral Parente, R.M.; Aguilar Martín, C. A new clinical decision support tool for improving the adequacy of anticoagulant therapy and reducing the incidence of stroke in nonvalvular atrial fibrillation. Medicine 2018, 97, e9578. [CrossRef]

32. Andreu-Cayuelas, J.M.; Marín, F.; Flores-Blanco, P.J.; García Alberola, A.; Manzano-Fernández, S. Methods of kidney function assessment in non-valvular atrial fibrillation in the era of new oral anticoagulants. Eur. J. Intern. Med. 2014, 25, e71-e72. [CrossRef] [PubMed]

33. Reilly, P.A.; Lehr, T.; Haertter, S.; Connolly, S.J.; Yusuf, S.; Eikelboom, J.W.; Ezekowitz, M.D.; Nehmiz, G.; Wang, S.; Wallentin, L. The effect of dabigatran plasma concentrations and patient characteristics on the frequency of ischemic stroke and major bleeding in atrial fibrillation patients: The RE-LY trial (Randomized Evaluation of Long-Term Anticoagulation Therapy). J. Am. Coll. Cardiol. 2014, 63, 321-328. [CrossRef] [PubMed]

34. Giner, M.; Vives, R.; Molina, A.; Morros, R.; Mena, D. Estudi D'utilització D'anticoagulants Orals en Fibril-Lació Auricular no Valvular; Servei Català de la Salut. Departament de Salut. Generalitat de Catalunya: Barcelona, Spain, 2019.

35. Ruff, C.T.; Giugliano, R.P.; Braunwald, E.; Hoffman, E.B.; Deenadayalu, N.; Ezekowitz, M.D.; Camm, A.J.; Weitz, J.I.; Lewis, B.S.; Parkhomenko, A.; et al. Comparison of the efficacy and safety of new oral anticoagulants with warfarin in patients with atrial fibrillation: A meta-analysis of randomised trials. Lancet 2014, 383, 955-962. [CrossRef] 